\title{
Zur Reindividualisierung der grundrechtsdogmatischen Systembildung
}

\author{
Ralf Müller-Terpitz*
}

Rezension zu Klaus Ferdinand Gärditz, Hochschulorganisation und verwaltungsrechtliche Systembildung, Tübingen (Mohr Siebeck - Jus Publicum, Band 182), 2009727 Seiten, $119 €$. ISBN 978-3-16-149958-6

1. Schon seit langem sind Hochschulen Gegenstand gesetzgeberischer Reformbestrebungen. Standen diese zu Beginn der 70er Jahre noch ganz im Zeichen einer Demokratisierung hochschulischer Bildungseinrichtungen von innen heraus, konzentriert sich der Gesetzgeber angesichts wachsender Studierendenzahlen bei konstant knappen finanziellen Ressourcen seit den 90er Jahren auf ihre allseitige Effizienzsteigerung. Dieses auf eine Erhöhung des „Lehr- und Forschungsoutputs“ gerichtete Ziel versucht der Gesetzgeber einerseits durch deregulative Maßnahmen, sprich durch die Stärkung hochschulischer Autonomie, andererseits durch eine Ökonomisierung und Wettbewerbsorientierung ihrer Denk- bzw. Handlungsmuster sowie - damit verbunden - durch eine Professionalisierung, Erneuerung und Stärkung ihrer Leitungsstrukturen zu erreichen. Zu diesem Zweck wurden die Präsidien bzw. Rektorate nicht nur kompetenziell aufgewertet, sondern auch mit externem Sachverstand besetzte entscheidungsbefugte Gremien - die Hochschulräte - institutionalisiert. Führungsstarke, gleichsam unternehmerisch agierende Hochschulen, so die dahinter stehende Idee, seien den Herausforderungen des 21. Jahrhunderts besser gewachsen, zumal wenn sie um externen, gleichfalls unternehmerisch orientierten Sachverstand ergänzt würden.

Vor der Folie dieses „New University Management“ setzt sich Klaus Ferdinand Gärditz mit Fragen einer verwaltungsrechtlichen Systembildung und ihrer Bedeutung für die Hochschulorganisation auseinander. Anders als beim Verfahrensrecht - so der Autor - bestehe im Organisationsrecht noch erheblicher Bedarf an einer solchen Systembildung. Das Hochschulorganisationsrecht fungiert für ihn insoweit als Referenzgebiet (S. 7 f., 43 f.). Parallel zum Verfahrensrecht sei der Verwaltungsorganisation dabei eine dienende Aufgabe für die Verwirklichung von Recht zuzuschreiben (S. 60 f.). Zudem kämen der Verwaltungsorganisation trotz ihrer starken problemund fachspezifischen Orientierung „stabile Grundfunktionen“ - nicht zuletzt für die rechtsstaatliche Legitimation verwaltungsrechtlicher Willensbildung sowie für den Grundrechtsschutz der von ihr Betroffenen - zu (S. 80 ff.).

Dogmatischer Ausgangspunkt für die Entfaltung eines Hochschulorganisationsrechts ist für den Autor die individual-abwehrrechtliche Funktion der Wissenschafts-

* Prof. Dr. Ralf Müller-Terpitz ist Inhaber des Lehrstuhls für Staats- und Verwaltungsrecht sowie Wirtschaftsverwaltungs-, Medien und Informationsrecht an der Juristischen Fakultät der Universität Passau. 
freiheit, an deren "grundrechtsdogmatischen Kern" sich andere, „derivative“ Grundrechtsfunktionen - gemeint sind die objektiv-rechtlichen Gehalte der Grundrechte in Gestalt organisations- und verfahrensrechtlicher Schutzaspekte - „nur ankristallisieren“ (S. 283 ff., 299). Gärditz spricht letzteren Grundrechtsdimensionen mithin lediglich einen „dienenden Charakter“ zu und erteilt ihrer „schleichenden Verselbstständigung vom Individuum als personalem Substrat liberaler Grundrechtskonzeption" eine deutliche Abfuhr (S. 329 f.). Dessen ungeachtet bleibe die abwehrrechtliche Dimension der Grundrechte für sich genommen unzureichend, um Freiheit und individuelle Selbstbestimmung zu gewährleisten (S. 337). Für das Hochschulorganisationsrecht zieht der Autor hieraus den Schluss, dass objektive Aussagen des Art. 5 Abs. 3 S. 1 GG über die binnenuniversitäre Organisation nicht Bestandteile einer objektiven Ordnungsidee seien, die den Grundrechtssubjekten gleichsam „übergestülpt“ werden könnten. Vielmehr seien sie die Konsequenz daraus, dass wissenschaftliche Tätigkeit im Rahmen staatlicher Organisation Grundrechtsschutz genieße, dessen verfassungsrechtlich gebotene Effektivität von einem „wissenschaftsadäquaten formalen Rahmen“ abhänge (S. 340). Zwar suche auch die Rechtsprechung des Bundesverfassungsgerichts die Lösung von Binnenkonflikten der Hochschulorganisation in der subjektiv-rechtlichen Seite der Wissenschaftsfreiheit. Dies geschehe indes nur „vordergründig“ und mit der Wirkung einer Rücknahme, „ja geradezu Ausdünnung “ des objektiv-rechtlich begründeten Grundrechtsschutzes durch Organisation und Verfahren zugunsten gesetzgeberischer Prognose- und Einschätzungsspielräume ( $\mathrm{S} .347 \mathrm{f}$.).

Untauglich zur verwaltungsrechtlichen Systembildung, welche Gärditz als genuin rechtswissenschaftlich-normative Aufgabe begreift (S. $100 \mathrm{ff}$.), seien hingegen die steuerungswissenschaftlichen Governance- und Netzwerkbegriffe. Sie hätten rein faktisch-soziologische Bedeutung und vertrügen sich nicht mit dem verfassungsrechtlichen Konzept demokratisch legitimierter Verantwortung und grundrechtlich gebotener Kontrolle öffentlicher Entscheidungsfindung (S. 216). Eine wissenschaftsadäquate Hochschulorganisation gründe sich vielmehr auf der individuellen Freiheit ihrer Mitglieder; ihre vornehmste Aufgabe sei es, diese Freiheit im Rahmen kollektiver Entscheidungen zu schützen. Vorrangiges Organisationsziel eines allgemeinen Hochschulorganisationsmodells sei deshalb mittelbarer Freiheitsschutz, nicht hingegen der Schutz der Institution als solcher (S. 439). Ungeachtet fortschreitender föderaler Pluralisierung und landesrechtlicher Deregulierung weise das Hochschulorganisationsrecht dabei stabile Grundprinzipien auf, zu denen Gärditz insbesondere das Repräsentations- und Kollegialprinzip sowie das Prinzip der Selbstorganschaft rechnet (S. 444 ff.). Das Repräsentationsprinzip sei nötig, um im Kontext wissenschaftlicher Heterogenität komplexe Entscheidungen fällen zu können, wobei Repräsentation die Freiheit des Einzelnen stabilisiere (S. 458 f.); freilich dürfe Letztere nicht dazu dienen, „wissenschaftliche Erkenntnisfragen (durch) Mehrheitsentscheidung“ 
zu determinieren (S. 460). Repräsentative Organisationsstrukturen müssten den Wissenschaftlern vielmehr ein hohes Maß an individueller Selbstbestimmung, etwa durch maßgebenden Einfluss in Fragen „wissenschaftlich-fachlicher Prägung“, erhalten (S. 467). Zusätzliche Absicherung erfahre die individuelle Freiheit in repräsentativen Entscheidungsstrukturen durch das verfassungsrechtlich verankerte Kollegialprinzip, welches zum Ausgleich und Kompromiss zwinge und somit rein hierarchisch getroffene, möglicherweise politisch motivierte Entscheidungen zu verhindern imstande sei. Es verwirkliche sich in fachlich kompetenten Organen wie dem Fakultätsrat und Senat, welche der Autor als „Basisorgane der Hochschule“ charakterisiert (S. 535). Auch das Prinzip der Selbstorganschaft sei verfassungsrechtlich geboten und stehe unkontrollierter Entscheidungskompetenz Externer - angesprochen sind hiermit die Hochschulräte - entgegen. Akademische Legitimation sei nur bei Entscheidung durch eigene, gewählte Organe möglich (S. 496 ff.).

Die Besonderheiten des Hochschulorganisationsrechts, das im Kern der Sicherung individueller Freiheitsentfaltung und struktureller Staatsferne diene, bringe es - so die Conclusio des Autors - mit sich, dass es nur bedingt als Vorbild für das allgemeine Verwaltungsorganisationsrecht taugt (S. 619 f.). Wohl aber könnten seine Eigenheiten als Kontrast, als „Eigenwert des Besonderen (...) Vergewisserung über allgemeine Dogmen“ liefern. Auch könnten sie in besonderer Weise den „Eigenwert des Formalen", d.h. den Umstand belegen, dass Organisationsrecht nicht nur als Instrument des materiellen Rechts, sondern auch als in formale Strukturen geronnener Interessenausgleich und Verteilungsschlüssel für Entscheidungsmacht und Entscheidungslegitimation zu begreifen sei (S. 620 f.).

2. Klaus Ferdinand Gärditz hat eine Habilitationsschrift vorgelegt, die durch ihren breiten thematischen Zugang, ihren Detailreichtum sowie ihre Gelehrsamkeit beeindruckt. Zwar verfehlt der Autor das selbst gesteckte Ziel ein Stück weit, da er den referenziellen Charakter des Hochschulrechts für das Organisationsrecht im Allgemeinen nur als Kontrapunkt herauszustellen vermag. Dessen ungeachtet hat er für organisationsrechtliche Fragen - seien es solche allgemeiner oder hochschulbezogener Art - eine „gedankliche Fundgrube“ ausgehoben, deren Inhalte und Schlussfolgerungen nicht samt und sonders geteilt zu werden brauchen, ${ }^{1}$ auf die man zur Vertiefung der behandelten Fragestellungen aber immer wieder zurückgreifen kann.

$\mathrm{Zu}$ diesen „gedanklichen Fundstücken“ gehören insbesondere die grundrechtsdogmatischen Ausführungen Gärditz', die in ihrer Grundsätzlichkeit über das von ihm untersuchte Thema hinausweisen. Zu Recht beschreibt Gärditz die individual-abwehrrechtliche Dimension der Grundrechte als deren „grundrechtsdogmatischen Kern“, an den sich andere, von ihm als „derivativ“ qualifizierte objektiv-rechtliche

1 Kritisch insoweit etwa Fehling, Die Verwaltung 43 (2010), 127: „Die differenzierte Argumentation mündet allerdings in (...) undifferenziert strukturkonservative Ergebnisse. (...).“. 
Grundrechtsfunktionen „,anzukristallisieren“ hätten. Spontan mag man meinen, dass Gärditz an dieser Stelle lediglich gefestigte Grundrechtsdogmatik, sprich alten Wein in neuen Schläuchen präsentiert. Das Bundesverfassungsgericht selbst hat in seiner hochschulorganisationsrechtlichen Leitentscheidung zum niedersächsischen Vorschaltgesetz diese dienende Funktion hervorgehoben, indem es den Gesetzgeber aufforderte, sein Handeln positiv an der in Art. 5 Abs. 3 GG verkörperten Wertentscheidung auszurichten, d.h. sich schützend und fördernd einer Aushöhlung der (individual-abwehrrechtlichen) Wissenschaftsfreiheit entgegen zu stemmen. ${ }^{2}$ Dem Autor ist jedoch beizupflichten, wenn er eine Abkehr vom „dienenden Charakter“ der objektiv-rechtlichen Grundrechtsgehalte hin zu einer „schleichenden Verselbstständigung vom Individuum als personalem Substrat liberaler Grundrechtskonzeption“ diagnostiziert. Diese Entwicklung ist nicht auf das von ihm untersuchte Hochschulorganisationsrecht beschränkt. Schon für lebenswissenschaftliche Kontexte hat sich der Rezensent kritisch mit Bestrebungen im rechtswissenschaftlichen Schrifttum auseinandergesetzt, die darauf abzielen, pränatales menschliches Leben und seinen Schutz vom personalen Substrat des Lebens- und Leibesgrundrechts (Art. 2 Abs. 2 S. 1 GG) zu entkoppeln und als rein objektiv-rechtliches Schutzgut zu apostrophieren. ${ }^{3}$ Die Stoßrichtung solcher „Verselbstständigungen“ objektiv-rechtlicher Grundrechtsgehalte ist in den Lebenswissenschaften eine ähnliche wie im Hochschulorganisationsrecht: Das sodann rein objektiv-rechtlich interpretierte Grundrecht wird nicht mehr als ein dem Individuum dienender subjektiver Anspruch (auf Unterlassung staatlicher Ingerenz oder auf staatlichen Schutz) verstanden, sondern als ein Gestaltungsauftrag an die Adresse des Gesetzgebers, welchem insoweit ein weiter Gestaltungs- und Abwägungsspielraum im Hinblick auf konfligierende Rechtsgüter oder anderweitige politische Interessen zugebilligt wird.

Die von Gärditz mit Verve vorgetragene und sich wie ein roter Faden durch seine Habilitationsschrift ziehende „Reindividualisierung der grundrechtsdogmatischen (...) Systembildung “ (S. 629) lässt sich zudem für ein weiteres Grundrecht außerhalb des Art. 5 Abs. 3 GG fruchtbar machen: die Rundfunkfreiheit. Die Parallelen der Rundfunk- und Wissenschaftsfreiheit sind frappant: In beiden Fällen verwirklicht sich grundrechtliche Freiheit im hohen Maße durch öffentlich-rechtliche Institutionen - hier die Rundfunkanstalten, dort die staatlichen Universitäten. Beide Institutionen sind jeweils dem Gemeinwohl verpflichtet und durch das Prinzip der Staatsferne gekennzeichnet. Von daher ist es kein Zufall, dass Gärditz an etlichen Stellen seiner Arbeit explizit auf den Rundfunkbereich Bezug nimmt. ${ }^{4}$ Trotz dieser Gemeinsamkeiten könnte die dogmatische Konstruktion beider Grundrechte kaum unter-

2 Vgl. BVerfGE 35, 79 (113).

3 Vgl. insoweit Müller-Terpitz, Der Schutz des pränatalen Lebens, Tübingen 2007, S. 149 ff., 169 ff., $270 \mathrm{ff}$.

4 Vgl. insoweit die Nachweise im Sachregister zum Stichwort „Rundfunk“ (S. 725). 
schiedlicher sein: Während das Bundesverfassungsgericht die Wissenschaftsfreiheit als klassische individualrechtliche Verbürgung begreift, dient die Rundfunkfreiheit nach seiner ständigen Rechtsprechung nicht zuvörderst der individuellen Persönlichkeitsentfaltung, sondern der freien Meinungsbildung. Der in Art. 5 Abs. 1 S. 2 GG enthaltene Auftrag zur Gewährleistung der Rundfunkfreiheit verlangt dem Gericht zufolge deshalb nach einer positiven Ordnung, die sicherstellt, dass die Vielfalt der bestehenden Meinungen im Rundfunk in möglichster Breite und Vollständigkeit Ausdruck findet. Die Ausgestaltung dieser Ordnung sei Aufgabe des Gesetzgebers, der dabei einen weiten Gestaltungsspielraum für sich in Anspruch nehmen könne. ${ }^{5}$

Wer wie Gärditz die Grundrechte in klassisch-liberaler Tradition streng individualabwehrrechtlich interpretiert, kann eine solche grundrechtliche Sonderdogmatik freilich nicht gutheißen. Denn mit „der fremdnützigen Instrumentalisierung des Grundrechts (d.i. die Rundfunkfreiheit - d. Verf.) wird die Definitionsmacht des Einzelnen letztlich zugunsten staatlicher Definition von Freiheitsinhalten jedenfalls partiell abgelöst“ (S. 290). Vor diesem Hintergrund verwundert es wenig, dass der Autor einer Ausgestaltungsdogmatik prinzipiell ablehnend gegenüber steht, da sich diese „nur begrenzt mit der Prämisse (verträgt), dass auch objektive Gewährleistungen an die individuellen Freiheitsrechte rückangebunden bleiben müssen “ (S. 336). Eine solche Kritik an der rundfunkrechtlichen Sonderdogmatik ist nicht neu, erhält durch Gärditz’ Schrift allerdings „Schützenhilfe“ aus anderer Warte - der Wissenschaftsfreiheit -, welche strukturverwandte Merkmale (starker Gemeinwohlbezug, staatsferne Tätigkeit) zu Ersterer aufweist.

Selbst wenn man die empirische Prämisse für diese rundfunkrechtliche Sonderdogmatik - die Breitenwirkung, Aktualität und Suggestivkraft des Rundfunks - teilt (was im Internetzeitalter keineswegs mehr eine Selbstverständlichkeit ist), bedarf es ihrer nicht. Hierauf ist von anderen Autoren, insbesondere von Karl-Eberhard Hain, bereits mehrfach überzeugend hingewiesen worden: Die Rundfunkfreiheit lässt sich als eine individual-abwerrechtliche Verbürgung denken, ohne dass dies zu substanziellen Verwerfungen für die gegenwärtige einfachgesetzliche Rundfunkordnung führte. Die Rundfunkgesetzgebung figurierte bei einer solch „klassischen“ Sichtweise nicht als Ausgestaltung des grundrechtlichen Schutzbereichs, sondern als Schranke im Sinne des Art. 5 Abs. 2 GG. Ihre Notwendigkeit und Legitimation bezöge sie einerseits aus dem Demokratieprinzip sowie aus anderen Verfassungsrechtsgütern, allen voran den Grundrechten; andererseits müsste sie sich am rationalitätsstiftenden Prinzip der Verhältnismäßigkeit messen lassen. Eine solche Sichtweise stünde nicht nur mit europäischem Rundfunkrecht im Einklang, sondern würde auch den nachgerade begriffsjuristischen Streit um den verfassungsrechtlichen Rundfunkbegriff entschärfen, da auch neuere publizistische Erscheinungsformen, wie beispielsweise journalistisch- 
redaktionell gestaltete Online-Medien, nicht mehr „Gefahr“ liefen, allein aufgrund ihrer Qualifikation als Rundfunk i.S. des Art. 5 Abs. 1 S. 2 GG in den Sog der verfassungsgerichtlichen Ausgestaltungsdogmatik zu geraten. ${ }^{6}$

Der Umstand, dass einem Grundrecht dienende Funktionen zukommen können, so lassen sich Gärditz' grundrechtsdogmatische Ausführungen insoweit resümieren, rechtfertigt für sich genommen noch keine Abkehr vom klassisch-liberalen Grundrechtsmodell. Solche dienenden Funktionen kommen nicht nur der Rundfunkfreiheit, sondern auch anderen grundrechtlichen Verbürgungen, etwa der Wissenschafts-, aber auch der Pressefreiheit, zu, ohne dass das Bundesverfassungsgericht für diese Freiheitsrechte bewährte Pfade der Grundrechtsdogmatik verlassen hätte. Die Arbeit von Gärditz schärft deshalb erneut den Blick für den Umstand, dass die bundesverfassungsgerichtliche Ausgestaltungsdogmatik für die Rundfunkfreiheit einen grundrechtsdogmatischen „Fremdkörper“ darstellt - und zwar nicht nur in Bezug auf die Kommunikationsgrundrechte des Art. 5 Abs. 1 GG, sondern auch in Bezug auf die anderen in Art. 5 GG statuierten, gleichfalls durch kommunikative Kontexte geprägten Grundrechte (Kunst- und Wissenschaftsfreiheit). Er liefert zugleich gute Argumente, warum es geboten erscheint, an dieser Sonderdogmatik nicht länger festzuhalten.

6 Vgl. zum Vorstehenden zuletzt und ausführlich Hain, in: Dörr (Hrsg.), Die Macht der Medien. Medienrechtliches Kolloquium zum 75. Geburtstag von Hartmut Schiedermair, Frankfurt a.M. u.a., 2011, S. 63 ff. m.w.N. 